\title{
A Novel p.G141R Mutation in ILDR1 Leads to Recessive Nonsyndromic Deafness DFNB42 in Two Chinese Han Families
}

\author{
Xueling Wang $\mathbb{D}^{1,2,3}$ Longhao Wang $\mathbb{D}^{1,2,3}$ Hu Peng $\mathbb{D}^{1,2,3,4}$ Tao Yang $\mathbb{D}^{1,2,3}$ \\ and Hao Wu id $1,2,3$ \\ ${ }^{1}$ Department of Otorhinolaryngology-Head and Neck Surgery, Shanghai Ninth People's Hospital, Shanghai Jiao Tong University \\ School of Medicine, Shanghai, China \\ ${ }^{2}$ Ear Institute, Shanghai Jiao Tong University School of Medicine, Shanghai, China \\ ${ }^{3}$ Shanghai Key Laboratory of Translational Medicine on Ear and Nose Diseases, Shanghai, China \\ ${ }^{4}$ Department of Otolaryngology-Head and Neck Surgery, Changzheng Hospital, Second Military Medical University, Shanghai, China
}

Correspondence should be addressed to Tao Yang; yangtfxl@sina.com and Hao Wu; wuhao622@sina.cn

Received 25 January 2018; Accepted 27 February 2018; Published 16 April 2018

Academic Editor: Renjie Chai

Copyright (C) 2018 Xueling Wang et al. This is an open access article distributed under the Creative Commons Attribution License, which permits unrestricted use, distribution, and reproduction in any medium, provided the original work is properly cited.

Genetic hearing impairment is highly heterogeneous. In this study, targeted next-generation sequencing (NGS) in two Chinese Han families identified a novel p.G141R homozygous mutation in ILDR1 as the genetic cause of the deafness. Consistent with the recessive inheritance, cosegregation of the p.G141R variant with the hearing loss was confirmed in members of both families by PCR amplification and Sanger sequencing. SNP genotyping analysis suggested that those two families were not closely related. Our study showed that targeted NGS is an effective tool for diagnosis of genetic deafness and that p.G141R in ILDR1 may be a relatively frequent mutation for DFNB42 in Chinese Hans.

\section{Introduction}

Approximately one in every thousand children suffers congenital or early-onset deafness. In more than half of such cases the hearing impairment can be attributed to genetic causes [1]. To date, more than 100 genes and 150 loci have been identified to be associated with nonsyndromic hearing impairment (Hereditary Hearing Loss Homepage) (http://hereditaryhearingloss.org), with approximately $80 \%$ of cases being autosomal recessive [2].

Mutations in the ILDR1 gene (OMIM 609739) lead to autosomal recessive nonsyndromic deafness DFNB42. ILDR1 encodes the immunoglobulin-like domain containing receptor 1 , a predicted type 1 transmembrane protein. It is widely expressed in a variety of tissues including prostate, testes, pancreas, and kidney. In 2011, Borck et al. first reported several loss-of-function mutations in ILDR1 resulting in autosomal recessive hearing impairment DFNB42 [3]. Ildr1 was found expressing in hair cells and supporting cells of the developing mouse cochlea and vestibule [3]. In Ildr1 knockout mice, hair cells undergo postnatal degeneration. At P35, all knockout mice had profound sensorineural hearing loss associated with a complete loss of outer hair cells and a disorganization of most stereocilia in inner hair cells [4]. Evidence suggested that IDLR1 functions as a water barrier at the tricellular tight junction [5].

To date, only a limited number (less than 20) of ILDRI mutations have been reported for DFNB42 in selected ethnic groups in Asia [3, 6-14]. In the present study, we identify a novel missense mutation in ILDR1 in two Chinese families with DFNB42.

\section{Materials and Methods}

2.1. Subjects and Clinical Evaluations. This study included two small Chinese recessive deaf families: family 1 (Figure 1(a)) and family 2 (Figure 1(b)). All affected individuals were clinically evaluated in the Department of Otolaryngology-Head and Neck Surgery, Shanghai Ninth People's Hospital, Shanghai, China. The evaluation included 


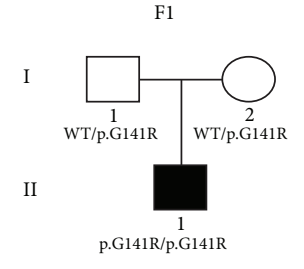

(a)

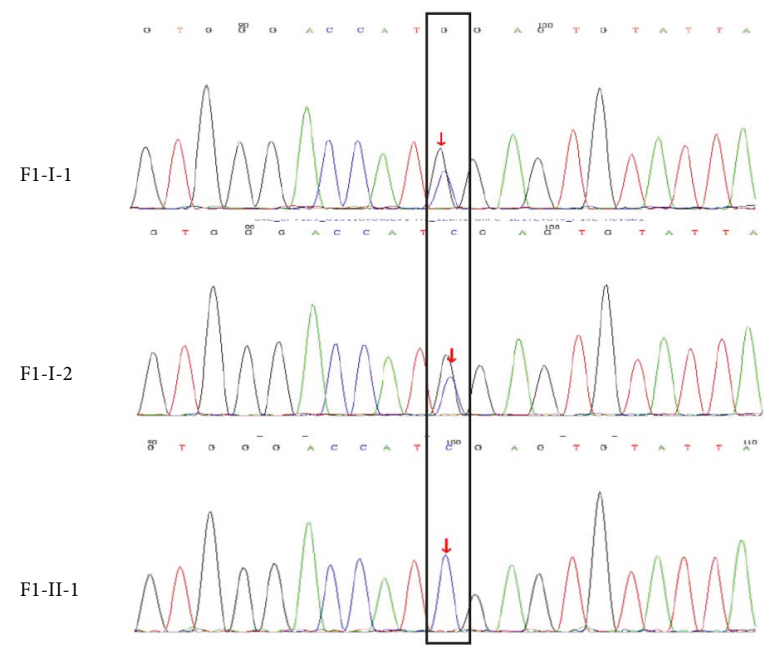

(c)

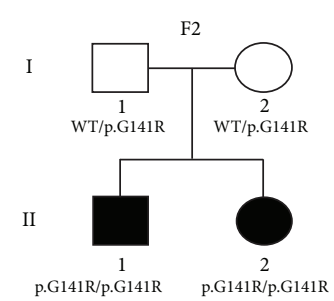

(b)

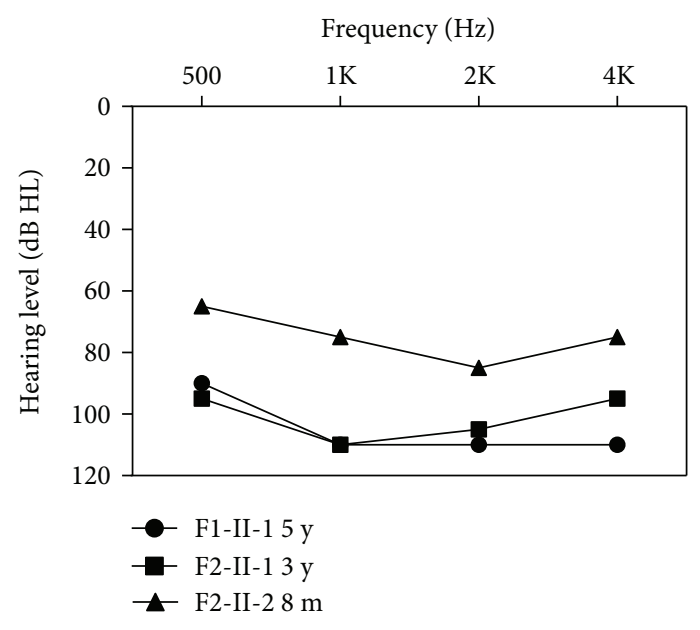

(d)

Figure 1: Pedigree and sequencing results of families F1 and F2. (a) Pedigree of family F1. (b) Pedigree of family F2. (c) Representative chromatograms showing the heterozygous (F1-I-1 and F1-I-2) and homozygous (F1-II-1) p.G141R mutation in ILDR1. (d) Audiograms of affected members.

a complete medical history interview and a detailed physical examination to rule out possible environmental or syndromic hearing impairment. Auditory evaluations were performed including otoscopic examination, otoacoustic emission (OAE), and pure tone audiometry (PTA). Hearing thresholds of subjects were determined by the air-conduction pure-tone average thresholds ranging from 250 to $8000 \mathrm{~Hz}$. Hearing level was classified as normal $(<20 \mathrm{~dB})$, mild $(20-40 \mathrm{~dB})$, moderate $(41-70 \mathrm{~dB})$, severe $(71-90 \mathrm{~dB})$, and profound $(>90 \mathrm{~dB})$. Hearing thresholds reported in this study were averages of the right and left ears. Romberg testing and tandem gait were performed for vestibular function examination. Computerized tomography (CT) scan of the temporal bone was carried out for excluding inner-ear anomalies.

2.2. Mutation Identification. Informed consent was obtained from all participants or from parents of the young subjects according to a protocol approved by the ethics committee of the Shanghai Ninth People's Hospital, Shanghai Jiao Tong University School of Medicine. Genomic DNA was extracted from peripheral blood samples using a blood DNA kit (TIANGEN Biotech Inc., Beijing, China). Targeted next-generation sequencing was performed in probands of family 1 and family 2 as previously described. Briefly, exon and flanking intron sequences of 159 known deafness genes (Supplementary Table 1) were captured by a customized capture array (NimbleGen, Roche) and sequenced on the Illumina HiSeq2000 analyzer. Data analysis and bioinformatics processing were performed following the standard operation procedure of Illumina. The minor allele frequencies (MAF) of the variants were determined using the gnomAD database (http://gnomad.broadinstitute.org/) and the in-house sequencing data of 200 Chinese Han normal hearing controls. Nonsynonymous variants with an MAF of 0.005 or less and consistent with the autosomal recessive inheritance were considered as candidate pathogenic mutations. Cosegregation of the disease phenotype and the detected variants were confirmed in all family members by PCR amplification and Sanger sequencing. Potential pathogenic effects of the candidate mutations were evaluated by the PolyPhen-2 [15], SIFT [16], and MutationTaster [17] programs.

\section{Results}

3.1. Clinical Features. For the three affected individuals in both families (F1-II-1, F2-II-1, and F2-II-2), the hearing impairment was prelingual and bilateral, and the severity ranged from moderate (F1-II-1) to profound (F2-II-1 and F2-II-2) (Figure 1(d)). Speech perception ability was severely impaired in patients F1-II-1 and F2-II-2. No predisposing factors were revealed from their medical and family history. All affected individuals failed the automated auditory brainstem response testing during the neonatal hearing screening and were diagnosed with sensorineural hearing loss. Hearing impairment appeared to be nonsyndromic, 


$\begin{array}{ll}\text { Homo sapiens } & \text { RADLVINEVMWWDHGVYYCTIEAPGDTSGDPDKEVKLIVLHWLTVIFII } \\ \text { Pongo abelii } & \text { RADLVINEVMWWDHGVYYCTIEAPGDTSGDPDKEVKLIVLHWLTVIFII } \\ \text { Papio anubis } & \text { RADLVINEVMWWDHGVYYCTIEAPGDTSGDPDKEVKLIVLHWLTVIFII } \\ \text { Chlorocebus sabaeus } & \text { RADLVINEVMWWHGVYYCTIEAPGDTSGDPDKEVKLIVLHWLTVIFII } \\ \text { Dipodomys ordii } & \text { RADLVINEVMWWHGVYYCTIEAPGDTSGDPDKEVKLIVLHWLTVIFII } \\ \text { Alligator sinensis } & \text { RADLVINEVMWWHGMYYCIVEAPGDTSGDPDKEVKLIVLHWLTVIFIV } \\ \text { Mus musculus } & \text { RADLVINEVMWWHGVYYCTIEAPGDTSGDPDKEVKLIVLHWLTVIFII } \\ \text { Xenopus laevis } & \text { RADLVISEVMWWHGVYFCSVEAQGDTSGDPDKEVKLVVLHWLTVLFII }\end{array}$

****** $\quad * * * * * * * * \quad * * \quad * * \quad * * * * * * * * * * * * * \quad * * * * * * * * * * *$

FIGURE 2: Multispecies sequence alignment of ILDR1 showing the evolutionarily conserved G141 residue.

TABLE 1: Genotype of SNPs in chromosome 3 for probands F1-II-1 and F2-II-1.

\begin{tabular}{|c|c|c|c|c|c|c|}
\hline \multirow{2}{*}{ dbSNP } & \multirow{2}{*}{ Chromosome } & \multirow{2}{*}{ Position } & \multirow{2}{*}{ Gene } & \multicolumn{2}{|c|}{ Genotype } & \multirow{2}{*}{ MAF in Chinese Hans } \\
\hline & & & & F1-II-1 & F2-II-1 & \\
\hline rs78962087 & $3 p 13$ & 70014199 & MITF & $\mathrm{C} / \mathrm{A}$ & $\mathrm{C} / \mathrm{C}$ & 0.0021 \\
\hline rs2306522 & $3 \mathrm{p} 21.31$ & 45542083 & LARS2 & $\mathrm{T} / \mathrm{T}$ & $\mathrm{T} / \mathrm{C}$ & 0.1413 \\
\hline rs11549809 & $3 \mathrm{p} 21.31$ & 45557707 & LARS2 & $\mathrm{G} / \mathrm{A}$ & $\mathrm{G} / \mathrm{G}$ & 0.1413 \\
\hline rs10578999 & $3 \mathrm{p} 21.31$ & 46751073 & TMIE & TAAG/T & $\mathrm{T} / \mathrm{T}$ & 0.7314 \\
\hline p.G141R & $3 q 13.33$ & 121720670 & ILDR1 & G/G & $\mathrm{G} / \mathrm{G}$ & 0 \\
\hline rs2877561 & $3 \mathrm{q} 13.33$ & 121712051 & $I L D R 1$ & $\mathrm{C} / \mathrm{C}$ & $\mathrm{A} / \mathrm{A}$ & 0.6304 \\
\hline rs16846663 & $3 \mathrm{q} 25.1$ & 150658264 & CLRN1 & G/A & G/G & 0 \\
\hline rs187218889 & $3 \mathrm{q} 25.1$ & 150690487 & CLRN1 & $\mathrm{G} / \mathrm{T}$ & $\mathrm{G} / \mathrm{G}$ & 0.0057 \\
\hline rs3796240 & $3 \mathrm{q} 25.1$ & 150690566 & CLRN1 & $\mathrm{T} / \mathrm{T}$ & $\mathrm{C} / \mathrm{C}$ & 0.0630 \\
\hline rs188384 & $3 q 28$ & 191074873 & CCDC50 & G/G & $\mathrm{C} / \mathrm{C}$ & 0.6435 \\
\hline rs11542549 & $3 \mathrm{q} 28$ & 191075902 & CCDC50 & $\mathrm{C} / \mathrm{T}$ & $\mathrm{C} / \mathrm{C}$ & 0.1652 \\
\hline rs2028573 & $3 \mathrm{q} 28$ & 191093080 & CCDC50 & $\mathrm{A} / \mathrm{A}$ & $\mathrm{G} / \mathrm{G}$ & 0.1500 \\
\hline rs4677728 & $3 q 28$ & 191093310 & CCDC50 & $\mathrm{A} / \mathrm{G}$ & $\mathrm{A} / \mathrm{A}$ & 0.0783 \\
\hline rs4677729 & $3 \mathrm{q} 28$ & 191093384 & CCDC50 & $\mathrm{A} / \mathrm{A}$ & $\mathrm{G} / \mathrm{G}$ & 0.0152 \\
\hline rs188384 & $3 q 28$ & 191094873 & CCDC50 & $\mathrm{G} / \mathrm{G}$ & $\mathrm{C} / \mathrm{C}$ & 0.6434 \\
\hline rs34031057 & $3 \mathrm{q} 28$ & 191097928 & CCDC50 & $\mathrm{G} / \mathrm{G}$ & G/GT & 0.0109 \\
\hline rs7624750 & $3 q 29$ & 193334991 & OPA1 & G/A & G/G & 0.2457 \\
\hline rs166850 & $3 q 29$ & 193355074 & OPA1 & $\mathrm{C} / \mathrm{C}$ & $\mathrm{T} / \mathrm{T}$ & 0.6739 \\
\hline rs10451941 & $3 q 29$ & 193355102 & OPA1 & $\mathrm{T} / \mathrm{C}$ & $\mathrm{T} / \mathrm{T}$ & 0.2000 \\
\hline rs9851685 & $3 q 29$ & 193374964 & OPA1 & $\mathrm{T} / \mathrm{C}$ & $\mathrm{T} / \mathrm{T}$ & 0.2326 \\
\hline rs3772393 & $3 q 29$ & 193336639 & OPA1 & $\mathrm{T} / \mathrm{C}$ & $\mathrm{T} / \mathrm{T}$ & 0.2000 \\
\hline
\end{tabular}

and no congenital anomalies, facial dysmorphisms, or intellectual disabilities were reported by the families or detected during physical examination. No vestibular symptoms were recorded.

3.2. Identification and Verification of the p.G141R Mutation in ILDR1. Targeted next-generation sequencing of 159 known deafness genes in probands F1-II-1 and F2-II-1 identified a total of 8 and 6 variants, respectively (Supplementary Table 2). In both cases, a homozygous c.421G>C (p.G141R) variant in ILDR1 (NM_001199799) was the only candidate variant consistent with the possible recessive inheritance. Sanger sequencing confirmed cosegregation of this mutation and the hearing impairment in family 1 and family 2, with all affected individuals being homozygous and all parents of the affected being heterozygous (Figure 1). The p.G141R mutation changed codon GGU to GCU in exon 4, substituting an evolutionarily conserved amino acid arginine with histidine in the immunoglobulin superfamily domain of ILDR1 (Figure 2). This mutation was predicted to be pathogenic by programs PolyPhen-2, SIFT, and MutationTaster. It has a minor allele frequency of 0.00009 in gnomAD and was not seen in 200 Chinese Han normal hearing controls. The F1 and F2 families originated from Jiangsu and Zhejiang provinces, respectively, two distinct regions of China. Neither of the families were from 
TABLE 2: Summary of mutations in ILDR1 that are associated with DFNB42.

\begin{tabular}{|c|c|c|c|c|}
\hline Mutation (protein) & Affected domains & Hearing phenotype & Ethnic group & Reference \\
\hline p.Met1Ileext+136 & Signal peptide and extracellular domain & Moderate to profound & Pakistan & [3] \\
\hline p.Gly20_Thr31del & Signal peptide and extracellular domain & Moderate to profound & Iranians & [3] \\
\hline p.V28SfsX31 & $\begin{array}{c}\text { Extracellular, transmembrane, and intracellular } \\
\text { domains }\end{array}$ & N/A & Pakistan & [3] \\
\hline p.Pro69His & Extracellular domain & $\begin{array}{c}\text { Postlingual onset and partial } \\
\text { deafness }\end{array}$ & Korean & {$[11]$} \\
\hline p.Arg97Gln & Extracellular domain & N/A & Pakistan & [3] \\
\hline p.Val102Glu & Extracellular domain & Severe to profound & Iranian & {$[10]$} \\
\hline p.Asn109_Pro111dup & Extracellular domain & Moderate to profound & Saudi Arabian & [9] \\
\hline p.Trp137CysfsX25 & Extracellular domain & N/A & Pakistan & [3] \\
\hline p.G141R & Extracellular domain & Moderate to profound & Chinese & $\begin{array}{l}\text { This } \\
\text { study }\end{array}$ \\
\hline p.Tyr143Cys & Extracellular domain & Moderate to profound & Iranians & {$[15]$} \\
\hline p.Trp168LysfsTer47 & Transmembrane and intracellular domains & Severe & Pakistan & [3] \\
\hline p.Gln195X & Intracellular domain & Severe to profound & Iranians & [3] \\
\hline p.Glu269ArgfsTer47 & Intracellular domain & Severe to profound & $\begin{array}{l}\text { United Arab } \\
\text { Emirates }\end{array}$ & [13] \\
\hline p.Q274X & Intracellular domain & N/A & Iranian & {$[8]$} \\
\hline p.C314X & Intracellular domain & N/A & Iranian & [7] \\
\hline p.Thr345ProfsX20 & Intracellular domain & Severe & Pakistan & [3] \\
\hline p.Glu379X & Intracellular domain & Severe to profound & Pakistan & [3] \\
\hline p.Glu394SerfsX15 & Intracellular domain & Severe & Pakistan & [3] \\
\hline p.S406X & Intracellular domain & Moderate to profound & Iranian & [10] \\
\hline p.Arg453Gln & Intracellular domain & Severe to profound & Pakistan & [3] \\
\hline
\end{tabular}

consanguineous marriage. Based on sequencing results of ILDR1 and other deafness genes in chromosome 3, F1-II-1 and F2-II-1 has a distinct genotype for a number of SNPs (Table 1), suggesting that those two probands were not closely related.

\section{Discussion}

In this study, we reported a novel p.G141R mutation in ILDR1 as the likely genetic cause for the autosomal recessive sensorineural hearing loss (ARSNHL) in two Chinese Han families. Evidence supporting the pathogenic roles of the p.G141R mutation includes: (1) Homozygous p.G141R mutation was identified in all three affected individuals (F1-II-1, F2-II-1, and F2-II-2), and the intrafamilial segregation pattern is consistent with the recessive inheritance (Figure 1). (2) Targeted NGS of 159 known deafness genes identified p.G141R as the only likely pathogenic mutation in probands of both families (Supplementary Table 2). (3) The p.G141R has an extremely low MAF of 0.00009 in 277,128 alleles in the gnomAD database and was absent in 200 ethnically matched normal hearing controls. (4) The p.G141R mutation changes an evolutionarily conserved amino of ILDR1 (Figure 2) and is unanimously predicted to be pathogenic by computational analysis tools PolyPhen-2, SIFT, and MutationTaster. (5) Based on the ACMG guideline [18], the p.G141R variant should be defined as likely pathogenic as it meets with 1 PS4 (the prevalence of the variant in affected individuals is significantly increased compared with the prevalence in controls) and 1 PM2 (extremely low frequency in controls if recessive) criteria.

To date, only 19 ILDR1 mutations have been reported. Interestingly, most mutations were identified from populations in south and west Asia (Table 2). To our knowledge, our study is the first report of ILDR1 mutations in Chinese Hans. The two families with the ILDR1 mutations were identified by targeted NGS of 162 sporadic deaf probands with likely recessive inheritance. The incidence of the pathogenic ILDR1 variants, therefore, is estimated to be approximately $1.2 \%$ in Chinese Han deaf patients. Since homozygous p.G141R mutations were identified in both nonconsanguineous families in the current study and our SNP genotyping analysis suggested that families F1 and F2 were unlikely to be closely related (Table 1), the p.G141R mutation in ILDR1 may be relatively frequent in Chinese Hans and deserve further screening in expanded studies of deaf patients in China.

Of the 19 previously reported ILDR1 mutations, the majority were nonsense mutations, indels, and stop-codon mutations that significantly truncates or alters the protein structure of ILDR1 (Figure 3, top). Interestingly, 5 of the 6 missense mutations in ILDR1 including p.G141R were within the extracellular immunoglobulin (Ig) superfamily domain (Figure 3, bottom), suggesting that this domain may have a critical role in relation to the hearing function. On the other hand, the onset and severity of hearing impairment associated with DFNB42 are diverse and there is no clear 


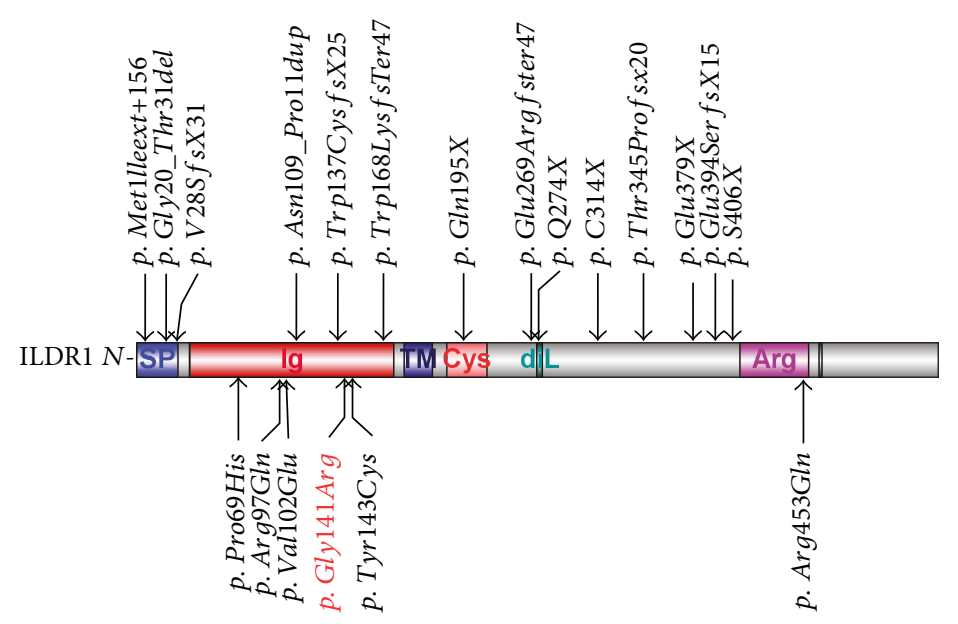

FIGURE 3: Schematic representation of the functional domains of ILDR1 and the locations of ILDR1 mutations. The nonmissense and missense mutations are listed above and under the domain structure, respectively.

genotype-phenotype correlation between the missense and nonmissense mutations (Table 2). Our results further verified this point as the affected individuals of families $\mathrm{F} 1$ and $\mathrm{F} 2 \mathrm{had}$ moderate and profound hearing impairment, respectively.

Our results also showed that targeted NGS is a powerful tool for the identification of the genetic causes of rare, heterogeneous disorders such as hearing impairment. The implication of this method should be recommended especially when the mutation is rare and the family size is limited, in which cases other methods such as an association study or linkage analysis will not be available.

\section{Conclusions}

The novel p.G141R mutation in ILDR1 is the likely genetic cause for the hearing impairment in two unrelated Chinese Han DFNB42 families. Targeted NGS is recommended for mutation identification of the rare deafness genes in small families or sporadic cases.

\section{Conflicts of Interest}

The authors declare no conflicts of interests.

\section{Authors' Contributions}

Xueling Wang, Longhao Wang, and Hu Peng contributed equally to this work.

\section{Acknowledgments}

This research was supported by grants from the National Key R\&D Program of China (2017YFC1001800 to HW), National Science Foundation of China (81700899 to XW, 81570930 to TY, and 81702643 to HP), Shanghai Municipal Science and Technology Commission (14DZ2260300 to HW and 16YF1403400 to HP), Shanghai Municipal Commission of Health and Family Planning Foundation (201740005 to XW and 201540173 to HP), China Postdoctoral Science Foundation (2017M611586 to HP) and Shanghai Municipal Education Commission-Gaofeng Clinical Medicine Grant (20152519 to TY).

\section{Supplementary Materials}

Supplementary Table 1: 159 deafness genes targeted for the next-generation sequencing. Supplementary Table 2: candidate variants identified by targeted NGS for probands F1-II-1 and F2-II-1. (Supplementary Materials)

\section{References}

[1] N. E. Morton, "Genetic epidemiology of hearing impairment," Annals of the New York Academy of Sciences, vol. 630, no. 1 Genetics of H, pp. 16-31, 1991.

[2] P. D'Adamo, V. I. Guerci, A. Fabretto et al., "Does epidermal thickening explain GJB2 high carrier frequency and heterozygote advantage?," European Journal of Human Genetics, vol. 17, no. 3, pp. 284-286, 2009.

[3] G. Borck, A. U. Rehman, K. Lee et al., "Loss-of-function mutations of ILDR1 cause autosomal-recessive hearing impairment DFNB42," American Journal of Human Genetics, vol. 88, no. 2, pp. 127-137, 2011.

[4] T. Higashi, T. Katsuno, S. Kitajiri, and M. Furuse, "Deficiency of Angulin-2/ILDR1, a tricellular tight junction-associated membrane protein, causes deafness with cochlear hair cell degeneration in mice," PLoS One, vol. 10, no. 3, article e0120674, 2015.

[5] Y. Gong, N. Himmerkus, A. Sunq et al., "ILDR1 is important for paracellular water transport and urine concentration mechanism," Proceedings of the National Academy of Sciences of the United States of America, vol. 114, no. 20, pp. 52715276, 2017.

[6] M. Aslam, M. Wajid, M. H. Chahrour et al., "A novel autosomal recessive nonsyndromic hearing impairment locus (DFNB42) maps to chromosome 3q13.31-q22.3," American Journal of Medical Genetics Part A, vol. 133A, no. 1, pp. 18-22, 2005.

[7] G. Bademci, J. Foster, N. Mahdieh et al., "Comprehensive analysis via exome sequencing uncovers genetic etiology in autosomal recessive nonsyndromic deafness in a large multiethnic cohort," Genetics in Medicine, vol. 18, no. 4, pp. 364-371, 2016. 
[8] O. Diaz-Horta, D. Duman, J. Foster et al., "Whole-exome sequencing efficiently detects rare mutations in autosomal recessive nonsyndromic hearing loss," PLoS One, vol. 7, no. 11, article e50628, 2012.

[9] K. Ramzan, K. Taibah, A. I. Tahir et al., "ILDR1: novel mutation and a rare cause of congenital deafness in the Saudi Arabian population," European Journal of Medical Genetics, vol. 57, no. 6, pp. 253-258, 2014.

[10] Z. Mehrjoo, M. Babanejad, K. Kahrizi, and H. Najmabadi, "Two novel mutations in ILDR1 gene cause autosomal recessive nonsyndromic hearing loss in consanguineous Iranian families," Journal of Genetics, vol. 94, no. 3, pp. 483-487, 2015.

[11] N. K. D. Kim, T. Higashi, K. Y. Lee et al., "Downsloping high-frequency hearing loss due to inner ear tricellular tight junction disruption by a novel ILDR1 mutation in the Iglike domain," PLoS One, vol. 10, no. 2, article e0116931, 2015.

[12] F. Talebi, F. G. Mardasi, J. M. Asl, and M. Sayahi, "Next-generation sequencing identifies three novel missense variants in ILDR1 and MYO6 genes in an Iranian family with hearing loss with review of the literature," International Journal of Pediatric Otorhinolaryngology, vol. 103, pp. 103-108, 2017.

[13] A. Tlili, A. Fahd al Mutery, M. Mahfood, W. Kamal Eddine Ahmad Mohamed, and K. Bajou, "Identification of a novel frameshift mutation in the ILDR1 gene in a UAE family, mutations review and phenotype genotype correlation," PLoS One, vol. 12, no. 9, article e0185281, 2017.

[14] M. Dokmanovic-chouinard, W. K. Chung, J. C. Chevre et al., "Positional cloning of "Lisch-Like", a candidate modifier of susceptibility to type 2 diabetes in mice," Plos Genetics, vol. 4, no. 7, p. e1000137, 2008

[15] I. A. Adzhubei, S. Schmidt, L. Peshkin et al., "A method and server for predicting damaging missense mutations," Nature Methods, vol. 7, no. 4, pp. 248-249, 2010.

[16] P. Kumar, S. Henikoff, and P. C. Ng, "Predicting the effects of coding non-synonymous variants on protein function using the SIFT algorithm," Nature Protocols, vol. 4, no. 7, pp. 1073-1081, 2009.

[17] J. M. Schwarz, D. N. Cooper, M. Schuelke, and D. Seelow, "MutationTaster2: mutation prediction for the deepsequencing age," Nature Methods, vol. 11, no. 4, pp. 361-362, 2014.

[18] S. Richards, N. Aziz, S. Bale et al., "Standards and guidelines for the interpretation of sequence variants: a joint consensus recommendation of the American College of Medical Genetics and Genomics and the Association for Molecular Pathology," Genetics in Medicine, vol. 5, no. 17, pp. 405-424, 2015. 


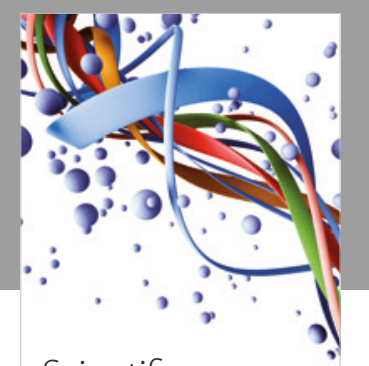

Scientifica
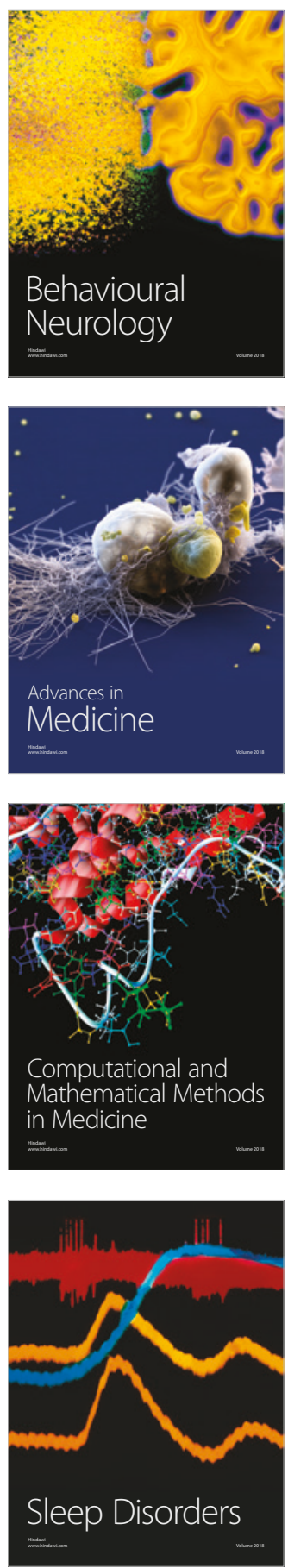

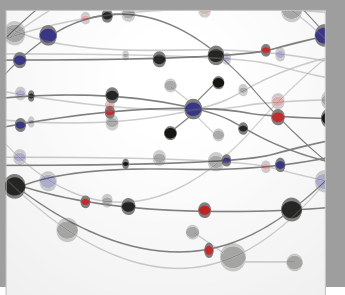

The Scientific World Journal

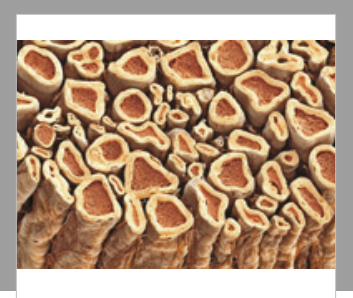

Case Reports in

Neurological Medicine

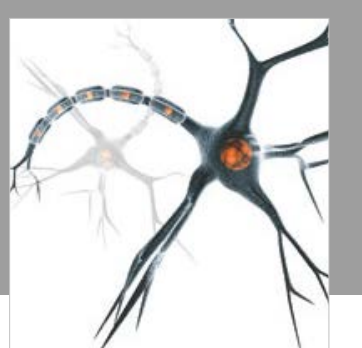

Neural Plasticity

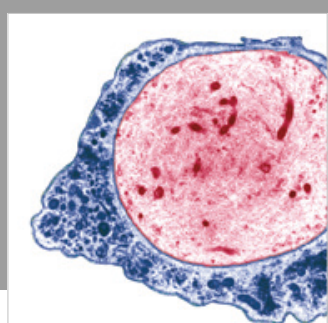

Multiple Sclerosis

International

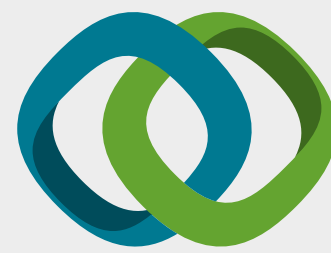

Hindawi

Submit your manuscripts at

www.hindawi.com
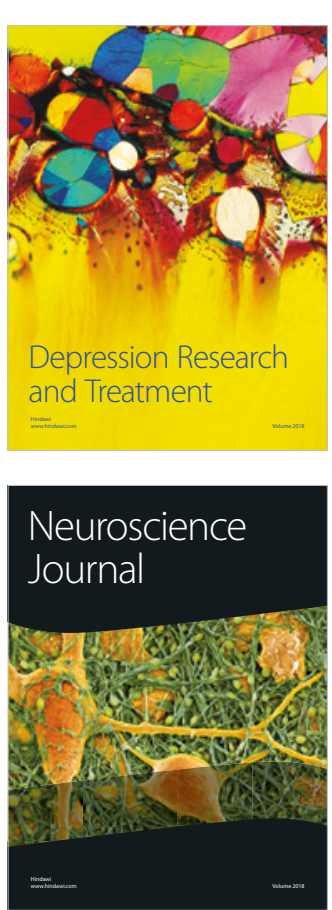

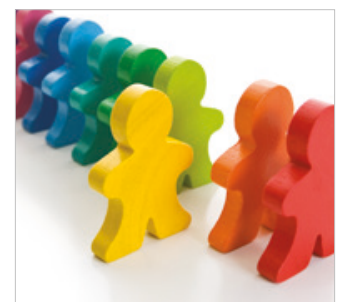

Autism

Research and Treatment
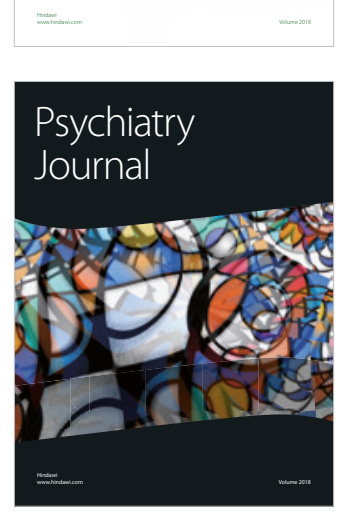
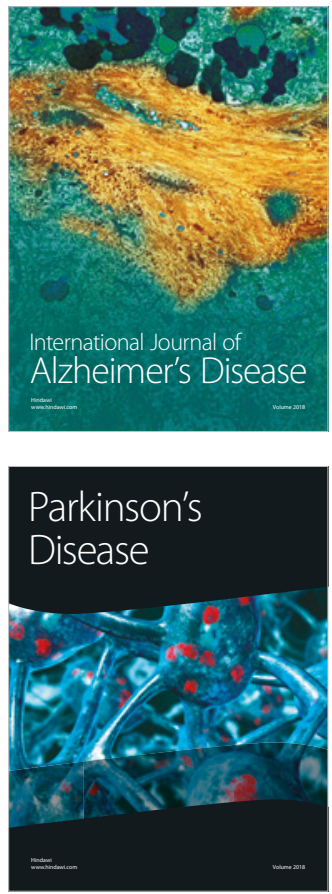
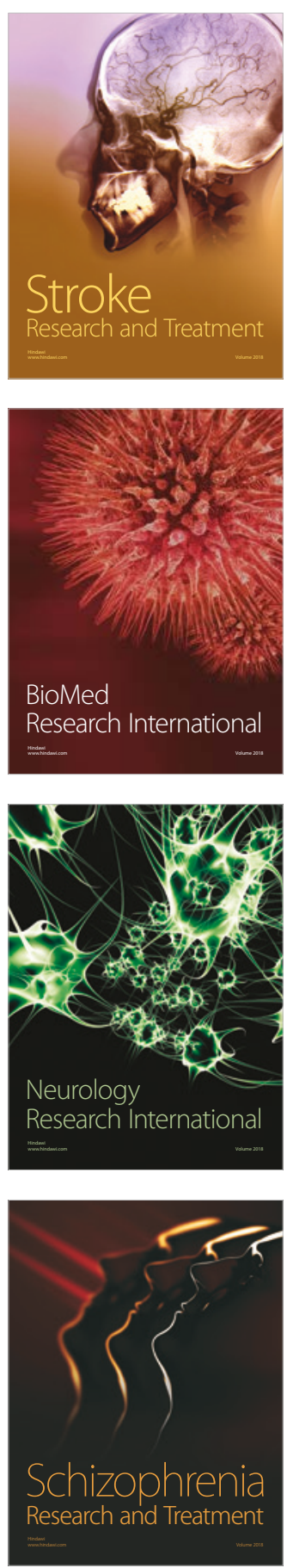